\title{
SENP3 Promotes Bladder Cancer Proliferation and EMT Transformation by Affecting the Expression of PYCR1 via DeSUMOylation of STAT3
}

\section{Zhuo Li}

Hunan Provincial People's Hospital

Jian Liu

Hunan Provincial People's Hospital

Huifeng Fu

Hunan Provincial People's Hospital

Yuanwei Li

Hunan Provincial People's Hospital

Qaing Lu

Hunan Provincial People's Hospital

Wei Song

Hunan Provincial People's Hospital

\section{Mingqiang Zeng ( $\sim$ l1787571894@163.com )}

Hunan Normal University First Hospital: Hunan Provincial People's Hospital https://orcid.org/00000002-7279-9646

\section{Research Article}

Keywords: Bladder Cancer, Signal Transducer and Activator of Transcription 3, SENP3, SUMOylation, Pyrroline-5-carboxylate Reductase 1, Epithelial Mesenchymal Transformation

Posted Date: September 17th, 2021

DOl: https://doi.org/10.21203/rs.3.rs-875422/v1

License: (a) (1) This work is licensed under a Creative Commons Attribution 4.0 International License. Read Full License 


\section{Abstract}

Abnormal activation of signal transducer and activator of transcription 3 (STAT3) has been found in various types of human cancers, including bladder cancer (BC). In our study, we examined the regulation of STAT3 post-translational modifications (PTMs) and found that SENP3 is high in bladder cancer. SENP3 and STAT3 were highly expressed in BC tissues when compared with tissue adjacent to carcinoma. SENP3 induced STAT3 protein level and p-STAT3 translocating into nuclear through deSUMOylation of STAT3. Further, nuclear STAT3, as a transcriptional activity factor, promoted pyrroline5-carboxylate reductase 1 PYCR1 gene and protein level by interacting with the promoter of (PYCR1). Next, we found that knockdown of PYCR1 inhibited Epithelial to mesenchymal transition of bladder cancer, and simultaneously mitigated the carcinogenic effects of STAT3. In vitro, STAT3 knockdown in bladder cancer cells inhibited cell proliferation, migration, and invasion. In contrast, SENP3 overexpression reversed these effects. In all, results lend novel insights into the regulation of STAT3, which has key roles in bladder cancer progression.

\section{Introduction}

Bladder cancer (BC), also known as urothelial cancer or urinary bladder cancer, is ranked 7th on the list of the most common cancers in men and the 17th in women worldwide. Unfortunately, the number of new cases continues to increase, especially in developed countries[1,2]. It was estimated that 550,000 people were diagnosed with bladder cancer, which accounts for $3 \%$ of all new cancer diagnoses[3]. The incidence of bladder cancer rises with age and is $3 \sim 4$ times higher in men. Cigarette smoking is the most important risk factor[4]. patients with advanced bladder cancer present with painless gross hematuria sometimes accompanied by the sensation of urgently needing to urinate.[5, 6]. In terms of survival outcomes, the five-year relative survival rates of advanced bladder cancer have seen little improvement in recent years[7]. According to WHO, the pathological types of bladder cancer include urothelial carcinoma, squamous cell carcinoma and adenocarcinoma of the bladder, among which the most common is urothelial carcinoma.

Signal transducer and activator of transcription 3(STAT3) is a highly regulated transcription factor that plays a prominent role in cellular growth and survival, as well as inflammation [8, 9]. MicroRNA miR-4500 inhibits STAT3 expression by targeting the STAT3 3'-3UTR region, STAT3 transcriptionally regulates CCR7 and promotes cancer[10]. Additionally, STAT3 signaling promotes resistance of prostate cancer to conventional chemoradiation and small-molecule inhibitors of other oncogenic signaling pathways[11]. The transcriptional activity of STAT3 is regulated by post-translational modifications (PTMs) in the protein[12]. For example, STAT3 is activated by phosphorylation of tyrosine 705 by upstream kinases, which leads to homodimerization, translocation to the nucleus, and induction of target gene expression[13,14]. Constitutive hyperactivation of STAT3 has been observed in multiple human cancers, where it contributes to tumor development. It has been reported that p-STAT3 induces methylation of ESR1 promoter in bladder cancer[15], and RacGAP1 induces phosphorylation of STAT3[16], which increases the expression of p-STAT3 and promotes its translocation into the nucleus to play a cancer- 
promoting role. Knocking-down of SENP3 greatly impaired basal and induced STAT3 phosphorylation by tobacco extract or interleukin[17]. Dysregulation has been identified in solid tumour and STAT3 activation is a marker for poor outcome[18]. The aim was to explore potential therapeutic strategies by targeting this pathway in BC.

SUMO modification is a reversible post-translational protein modification that is used in a wide spectrum of cellular processes. SUMOylation occurs when proteins are modified with the small-molecule proteins SUM01, SUMO2, or SUMO3[19, 20]. The modified substrates can be de-conjugated by a SUMO-specific protease from the SUMO-specific protease (SENP) family, in a process called de-SUMOylation. Seven SENPs (SENP1, 2, 3, 5, 6, 7, and 8) have been reported in mammals[19, 21]. Dynamics of SUMO and deSUMO modifications result in the alteration of protein activity, localization, and protein-protein or proteinDNA interaction, consequently regulating cellular behavior[22,23]. Senp3 is widely involved in a variety of diseases, including tumors, and has not been reported in bladder cancer[24]. SENP3 as a critical positive modulator of tobacco- or cytokine-induced STAT3 activation by promoting deSUMOylation of STAT3[16]. Here, we demonstrated deSUMOylation STAT3 by SENP3, which was activated to accumulated nuclear. However, there was little evidence for the association between SUMOylation and bladder cancer proliferation and epithelial mesenchymal transformation (EMT).

STAT3 in the nucleus upregulated the expression of oncogene PYCR1, which promoted bladder cancer proliferation and EMT. Pyrroline-5-carboxylate reductase 1 (PYCR1) is an enzyme involved in cell metabolism and is upregulated in cancer[25]. PYCR1 functions as an oncogene in bladder cancer. In addition, PYCR1 has been found to be highly expressed in a variety of malignancies, including bladder cancer, breast cancer, renal cell cancer and lung cancer[25-28]. Previous study showed that PYCR1 functions as an oncogene with poor prognosis in bladder cancer. Knockdown of PYCR1 inhibited the proliferation and invasion of bladder cancer cell lines.

The aim of the present study was to explore the effect and mechanism of STAT3 and its activation in bladder cancer. Our results demonstrated that SENP3 could enhance STAT3 phosphorylation and pSTAT3 distribution of nuclear through de-conjugating SUMO modification of STAT3. Furthermore, STAT3 promoted PYCR1 gene and protein level by interacting with its promoter. Lastly, Knockdown of PYCR1 inhibited Epithelial to mesenchymal transition of bladder cancer, and simultaneously mitigated the carcinogenic effects of STAT3.

\section{Materials And Methods}

\section{Cell culture}

the resuscitated cells were gently shaken and melted in a water bath at $37^{\circ} \mathrm{C}$ and transferred into a centrifuge tube containing $10 \mathrm{ml}$ dulbecco's modified eagle medium (DMEM); Centrifuge at $1000 \mathrm{rpm} / \mathrm{min}$ for $5 \mathrm{~min}$, pour out the supernatant, add medium to the centrifuge tube and suspend it, then transfer it to the culture flask; The cells were proliferated to $80 \%$ density for passage. 


\section{Fluorescence staining}

Cell slides were dipped and washed with PBS; which was fixed with $4 \%$ paraformaldehyde for $15 \mathrm{~min}$ at room temperature. Fixed cells were permeabilize with $0.5 \%$ TritonX-100 for $20 \mathrm{~min}$ at room temperature; The Cell slides were blocked at room temperature for $30 \mathrm{~min}$, and incubated with primary antibody at $4{ }^{\circ} \mathrm{C}$ overnight, followed by anti-rabbit secondary antibody conjugated with Alexa Flour 488 at room temperature for $1 \mathrm{~h}$. DAPI was used to stain the core and seal the film, and the images were observed and collected.

\section{Quantitative real-time PCR (RT-PCR)}

Preparation of cDNA, amplification of the target gene and preparation of RT-PCR reaction solution: $2 \times$ Master mix 10.0 (ul), Forward Primer 1.0 (ul), Reverse Primer 1.0 (ul), nuclease-free H2O 1.0 (ul), Total per Reaction 18.0 (ul); The PCR reaction solution was divided into eight PCR tubes and 2ul cDNA prepared was added. The difference of gene expression was detected by fluorescence quantitative PCR.

\section{Western Blot assay}

Cells were cultured to the density reached $80 \%$, cells were collected and resuspended with an appropriate volume of RIPA lysate for 30 min on ice. Pyrolysis liquid centrifuged with $13000 \mathrm{rpm}$ at $4^{\circ} \mathrm{C}$ for $20 \mathrm{~min}$, the supernatant was determined by BCA protein assay kit and diluted to the same concentration. The different treated samples with 30ug total protein content were separated on SDS-PAGE and transferred to PVDF membrane. Protein expressions were blocked with $5 \%$ skim milk for $1 \mathrm{~h}$ at room temperature. Primary antibody, diluted in the blocking solution, was incubate overnight at $4^{\circ} \mathrm{C}$; and the secondary antibody was for $1 \mathrm{~h}$ at room temperature. Finally, the membranes were visualized using Pierce ${ }^{\circledR} \mathrm{ECL}$ Western Blotting. Primary antibody: STAT3

(Abcam, ab32500), p-STAT3(Abcam, ab76315), FN(promab, 30506), E-cad(Ptgcn, 20874-1-AP), SENP3(Ptgcn,. 17659-1-AP), Sumo 2(Abcam, ab233222), PYCR1 (Ptgcn,. 66510-1-lg), $\beta$-actin(Ptgcn, 66009-1-lg).

\section{Trans well}

Cells were treated with drugs (or not) for a certain time. Add the serum culture medium containing $20 \%$ FBS into wells (600ul/well). Put trans well chambers in 24-well plates. Digest cells and resuspend cells with serum-free culture medium. Cell concentration should be determined according to specific conditions. Add cell suspension into trans well chambers (400ul/chamber). Put it in an incubator for a certain period of time. Determine the time by checking the literature. Take trans well chambers out and wipe cells that have not passed through the inside of the microporous filter with a cotton swab. Fix cells methanol with for 5 minutes. Dye cells with purple crystal and clean trans well chambers with pure water.

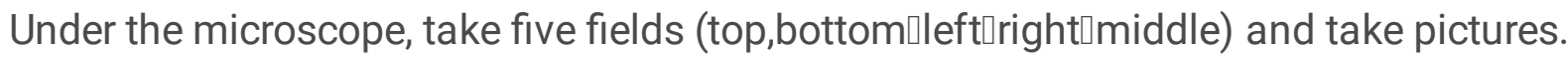


Tissue samples were deparaffinized at $60^{\circ} \mathrm{C}$ for half an hour,then deparaffinized and rehydrated. Antigen retrieval were performed in antigen Repair Solution along the microwave program. Tissue were permeabilized with $0.5 \%$ Triton X-100 in PBS for $20 \mathrm{~min}$ at room temperature, then shaking for 30 minutes with $1 \% \mathrm{H} 2 \mathrm{O} 2$. Blocking buffer $(0.5 \% \mathrm{BSA}+5 \%$ goat serum) was used to block for $30 \mathrm{~min}$ at room temperature. Primary Ab, diluted in blocking buffer (1:100 or 1:200), was incubated for overnight at $4^{\circ} \mathrm{C}$. Secondary Ab (Biotin-labeled secondary antibody, 1:200, diluted in blocking buffer) was incubated for $30 \mathrm{~min}$ at room temperature. $\mathrm{ABC}$ dilution $(1 \mathrm{mIPBS}+10 \mathrm{ul} \mathrm{A}+10 \mathrm{ulB})$ were configured half an hour. $\mathrm{DAB}$ Chromogenic solution were used for visualization.

\section{Extraction of plasma/nucleoprotein}

NucBuster TM Protein Exaction Kit (merckmillipore, 71183-3) was used to extract plasma/nucleoprotein as the flowing method. The collected cells were added with liquid nitrogen and quickly ground into a powder. Appropriate amount of powder were diluted with 150 ul NucBuster Reagent 1, then vortexed with high speed for $15 \mathrm{~S}$. The samples were placed on ice for $5 \mathrm{~min}$ and centrifuged with $16000 \mathrm{~g}$ at $4^{\circ} \mathrm{C}$ for $20 \mathrm{~min}$. The supernatant was served as cytoplasmic protein, and floccule was added $1 \mathrm{ul} 100 \times$ Protease Inhibitor Coctail, $1 \mathrm{ul} 100 \mathrm{mM}$ DTT and 75ul NucBuster Exaction Reagent. The mixture was vortexed with high speed for $15 \mathrm{~S}$ and placed on ice for $5 \mathrm{~min}$. Then the mixture centrifuged with $16000 \mathrm{~g}$ at $4^{\circ} \mathrm{C}$ for $20 \mathrm{~min}$. Cytoplasmic protein and nucleoprotein can be used immediately or stored in separate packages at $-80^{\circ} \mathrm{C}$.

\section{Transfection}

Seed $5 \times 10^{4}$ cells on 6 -well plates to achieve $70-90 \%$ cell convergence within 24 hours. Prepare the overexpressed plasmid-Lipofectamine ${ }^{\text {TM }} 3000$ complex as follows: Dilute 7.5ul Lipofectamine ${ }^{\text {TM }} 3000$ with 125ul Opti-Mem ${ }^{\circledR}$ I and mix gently; Dilute 5ul plasmid with 125ul Opti-Mem ${ }^{\circledR}$ I, then add 5ul P3000TM reagent, and mix gently; Mix the diluted plasmid with the diluted Lipofectamine ${ }^{\mathrm{TM}} 3000$, mix gently and incubate at room temperature for $5 \mathrm{~min}$ to allow the formation of the complex. Add plasmid-

LipofectaminetM3000 complex to each well containing the cells and medium. Incubate for 3-5 hours for downstream experiments.

\section{CCK8 assay}

assay was applied to determine the effects of test reagents on cell growth. Briefly, cells were

seeded in a 96-well plate with a density of $1 \times 10^{5}$ cells per well. After $24 \mathrm{~h}$, cells were treated with agents for indicated time. Then, $10 \mu \mathrm{L}$ of MTT solution was added to each well and incubated for $1 \mathrm{~h}$. 96-well were shake on the shaker for one minute. Finally, the absorbance was read at $550 \mathrm{~nm}$ using a microplate reader (Thermo Fisher Scientific, Franklin, MA, USA). Cell growth inhibitory ratio (\%) was calculated as following formula: Cell proliferation rate $\%=(O D$ value of test well $-O D$ value of background control well $) /($ OD value of control cell - OD value of background control) $\times 100 \%$. 
The cells were fixed in $1 \%$ formaldehyde and incubated at room temperature for 10 minutes. $1 \mathrm{~mL}$ of $10 \mathrm{X}$ Glycine was added to each dish to quench unreacted formaldehyde and incubate at room temperature for 5 minutes. These dishes were placed on ice. Medium was removed as much as possible. Each dish was added $2 \mathrm{~mL}$ cold PBS containing Protease Inhibitor Cocktail. The cells were collected with $800 \mathrm{~g}$ at $4^{\circ} \mathrm{C}$ for 5 minutes. During spin, each sample was combined with $0.5 \mathrm{~mL}$ of cell Lysis Buffer with $2.5 \mu \mathrm{L}$ of Protease Inhibitor Cocktail II.

\section{Tumor formation assay in nude mice}

Male BALB/c nude mice (6-week-old) were purchased from model animal research center of nanjing university and maintained in pathogen-free conditions. The mice were injected subcutaneously into the right flanks with $1 \times 106$ cells $/ \mathrm{mL}(0.1 \mathrm{~mL})$ of T24 cells, which were stably transfected with pLentiCon/NC, plentiSENP3/OE, plentiSENP3 + si-STAT3/OE + si-STAT3 plasmid and siRNA. Tumor growth was examined every 7 days, and tumor volumes were calculated using the equation, $V=0.52 \times$ length $\times$ width2. The tumor growth curve was plotted with time as the abscissa and tumor volume as the ordinate. Finally, each tumor was examined by immunohistochemical analysis. All animal care and experiments were conducted in accordance with national and institutional policies for animal health and well-being. The protocol was approved by the Institutional Animal Care and Use Committee.

\section{Statistical Analysis}

Sample size was determined upon availability, and chosen based on previous experience and previous studies. No exclusion criteria were pre-established. Samples or animals were randomly allocated among groups. Investigators were not blinded to group allocation during data collection and analyzes. No data were excluded. All statistical tests were performed with SigmaPlot 14.0. Data shown were presented by means \pm Standard Deviation (S.D.) from triplicate experiments performed in a parallel manner unless other wise indicated. Statistical significance was performed using an unpaired two-tailed Student's t test for two data sets when the data met the normal distribution tested by F-test. The variance between comparison groups was not similar. Detailed descriptions of all methods are provided in Supplementary Materials and Methods.

\section{Results}

\section{SENP3 protein level correlates with protein expression of STAT3 and p-STAT3 in bladder cancer}

The GEPIA database of bladder cancer showed that STAT3 gene expression in bladder cancer tissues was lower than that in adjacent normal tissues (Fig. 1A). However, survival curve analysis showed that the prognosis of patients with high STAT3 expression was poor (Fig. 1B). The contrast between gene expression level and gene function suggested that STAT3 might have post-translational protein modification. STAT3 has been reported to be sumoylation in tumors[16], and SENP3 expression in 
bladder cancer tissue was higher than that in adjacent or normal tissue (Fig. 1C). To address the relationship between SENP3 and STAT3 in bladder cancer, SENP3 and STAT3 proteins were detected in 8 cases of bladder cancer and para-cancerous tissues. The results showed that the expression levels of SENP3, STAT3 and p-STAT3 in bladder cancer tissues were higher than para-cancerous normal tissues (Fig. 1E). At the same time, we detected STAT3 and p-STAT3 in bladder cancer and para-cancerous normal tissues by IHC assay. The protein level of STAT3 and p-STAT3 in bladder cancer tissues was higher than that in normal tissues, especially the strong staining signal of p-STAT3 in the nucleus (Fig. 1F). In order to further detect the regulation of STAT3 and p-STAT3 by SENP3, two cell lines (T24 cells and EJ cells) with positive correlation between SENP3 and p-STAT3 proteins were selected for subsequent experiments (Fig. 1G). Taken together, SENP3 protein level is up-regulated in bladder cancer, which is correlates with protein expression of STAT3 and p-STAT3.

\section{SENP3 promotes proliferation and deSUMOylation of STAT3}

To further explore the mechanism of STAT3 regulated by SENP3, we constructed SENP3 stably knockdown and overexpression T24 cells and EJ cells. Both CCK8 assay and EDU immunofluorescence staining showed that overexpression of SENP3 promoted cell proliferation (Fig. 2A and Fig. 2C). Trans well staining indicated that SENP3 promoted cell invasion (Fig. 2B). To explore regulated-effect of STAT3 by overexpression and knockdown of SENP3 in T24 cells and EJ cells, We detected gene and protein levels of STAT3. SENP3 up-regulated the STAT3 protein level, not affect the gene expression of STAT3 (Fig. 2E and Fig. 2F). Meanwhile, SENP3 promoted the phosphorylation of STAT3 (Fig. 2E). SENP3 could enhance STAT3 phosphorylation by de-conjugating the SUMO2/3 modification of STAT3 in head and neck cancer[16]. To test whether SENP3 de-conjugates the SUMO modification of STAT3 and how to regulate it, immunoprecipitation assay suggested that SENP3 physically associates with STAT3 (Fig. 2G). When SENP3 binding to STAT3, STAT3-sumoylation level significantly decreased. To investigate the stability of desumoylated-STAT3 regulated by SENP3, treating with Cycloheximide (CHX) and MG132, overexpression of SENP3 delayed the degradation of STAT3, while knock-down of SENP3 accelerated the degradation of STAT3 (Fig. 2H). SENP3 improved the protein stability of STAT3. These results indicate that SENP3 promotes proliferation and deSUMOylates to stabilize STAT3 protein level.

\section{STAT3 mitigates the cancer-promoting effect of SENP3}

SENP3 is a cancer-promoting protein, which up-regulates protein level of STAT3. We test whether silencing STAT3 reverses the cancer-promoting effect of SENP3. When knocking down STAT3 in overexpression of SENP3 cells, cell proliferation and EDU positive staining were up-regulated, which closing to or lower than control group (Fig. 3A and Fig. 3F). Trans well staining indicated that Knockingdown of SENP3 clearly inhibited cell invasion comparing with overexpression of SENP3 (Fig. 3B and Fig. 3C), knock-down of STAT3 significantly reduced p-STAT3 protein level. Overexpression of SENP3 promoted EMT by reducing E-Ca and increasing FN protein levels. When knocking down STAT3, the changes of E-Ca and FN were reversed (Fig. 3E). These data suggest that STAT3 mitigates the cancerpromoting effect of overexpression of SENP3. 
STAT3 promotes gene and protein levels of PYCR1 by binding to promoter of PYCR1.

STAT3 in the nucleus performs transcriptional activation of the target gene. Studies have shown that PYCR1 acts as an oncogene function and is highly expressed in bladder cancer. To investigate the specific regulatory mechanism of STAT3 on PYCR1, we constructed STAT3 stably overexpression and knockdown cells. STAT3 promotes the gene and protein expression levels of PYCR1 (Fig. 4A and Fig. 4C). We predicted that STAT3 could potentially bind to the PyCR1 promoter region by using the Jaspar transcriptional interaction website, and the results of immunoprecipitation experiment showed that overexpression of STAT3 could increase the enrichment of STAT3 protein to the PyCR1 promoter region (Fig. 4B). DNA-affinity Precipitation Assay (DAPA) results show that PYCR1 promoter binds to the STAT3 protein (Fig. 4D). These results indicate that STAT3 promotes gene and protein levels of PYCR1 by binding to promoter of PYCR1.

\section{PYCR1 mitigates the carcinogenic effect of STAT3}

To test whether knock-down of PYCR1 reduces the carcinogenic effect of STAT3, we transfected with PYCR1 siRNA in overexpression of STAT3 cells. Compared with normal cells, overexpression of STAT3 promoted cell proliferation, invasion and EDU proliferation. However, silencing PYCR1 in overexpression of STAT3 cells completely rescued the cell proliferation and invasion to normal cells (Fig. 5A and Fig. 5F). Simultaneously, p-STAT3 protein level was obviously lower. STAT3 reduced E-Ca and increased FN protein levels. When knocking down PYCR1 of overexpression of STAT3 cells, the changes of E-Ca and FN could be reversed (Fig. 5E). Together, PYCR1 mitigates the carcinogenic effect of STAT3.

\section{SENP3 promotes tumor proliferation by upregulating STAT3 in vivo}

To determine the functional significance of SENP3, a subcutaneous transplantation tumor model in nude $\mathrm{BALB} / \mathrm{c}$ mice was established using the human bladder cancer cell line T24. The mice were divided into NC, SENP3(OE) and SENP3(OE) + si-STAT3 groups with 5 mice per group. The growth curve of tumor volumes showed that overexpression of SENP3 accelerated tumor growth compared with control group (Fig. 6A and Fig. 6B). However, knocking down STAT3 reduced tumor growth (Fig. 6A and Fig. 6B). The PCNA staining showed that knocking down STAT3 inhibited cell proliferation promoted by SENP3 (Fig. 6C). SENP3 upregulated PYCR1 protein level and promoted EMT transformation, but silencing STAT3 reversed the effect of SENP3 (Fig. 6D). Taken together, SENP3 promotes bladder cancer proliferation and EMT transformation by regulating STAT3.

\section{Discussion}

$\mathrm{BC}$ is the most common cancer of the urinary tract with 430,000 new cases and 165,000 deaths per year worldwide[29]. Unfortunately, the number of new cases continues to increase, which was estimated that 550,000 people were diagnosed with bladder cancer $[3,30]$. Although men are 4 times more likely than women to be diagnosed with BC, women often present with more advanced disease and have 
unfavorable prognosis[31]. To enhance bladder cancer therapy, new molecular targets for its diagnosis and prognosis must be identified, and new treatments must be developed.

The regulation of STAT3 transcriptional activity depends mainly on post-translational modifications (PTMs). Phosphorylation at Y705 or S727 is a crucial PTM that triggers STAT3 dimerization, nuclear translocation and maximal transactivation upon cytokine/growth factor stimulation[32]. however, many other PTMs, including acetylation, methylation, poly- and mono-ubiquitination, are required for STAT3 activation under various contexts[33-37]. SUMOylation, that is, the conjugation of SUMO1 or $2 / 3$ to the substrates, is an important PTM. SUMO2 and SUMO3 are closely related, referred to as SUMO2/3, whereas SUMO1 shares only partial similarity with SUMO2/3[38]. It have been reported to regulate their transcriptional activity negatively[39]. SUMOylation of STAT3 negatively regulates its activity by restraining Y705 phosphorylation in the nucleus in head and neck cancer[16]. These studies indicated that SUMOylation of STAT3 may play a key role in tumor development.

As a member of the SUMO-specific protease family, the function of SENP3 is known to regulate deSUMOylation of chromosome-associated proteins and chromosome stability in multiple cellular biological processes[36, 40]. Growing evidence has confirmed that SENP3 is highly expressed in some types of malignant tumors, such as head and neck cancer[16], ovarian cancer[41], and gastric cancer[40]. Some studies showed that SENP3 protects H9C2 cells from apoptosis triggered STAT3 pathway[17]. Our findings are consistent with those of previous studies. Here, we firstly report that SENP3 in bladder cancer tissues were higher than those in normal tissues. Previous published research results showed that STAT3 was highly expressed in bladder cancer and bladder cancer cell lines. Therefore, we further found that SENP3 could promote the proliferation of bladder cancer cells. Additionally, SENP3 was significantly correlated with STAT3 protein upregulation, not influent gene expression, suggesting that SENP3 may regulated STAT3-SUMOylation level. Further results indicated that SENP3 advanced STAT3 protein level and stability by facilitating STAT3-deSUMOylation. Furthermore, SENP3 significantly promoted the pSTAT3 protein expression, and especially distribution of p-STAT3 in the nucleus. Knock-down of STAT3 distinctly promoted the proliferation, migration, and invasion of bladder cancer. Functionally, it could reduce the carcinogenic effect of SENP3.

The pyrroline-5-carboxylate reductase (PYCR) family is a class of enzymes that play a key catalytic role in proline biosynthesis, which is mostly found in mitochondria and catalyzes proline synthesis and participates in cell metabolism[42, 43]. In addition, PYCR1 has been found to be highly expressed in a variety of malignancies, highly expressed in bladder cancer and knockdown of PYCR1 exerts a remarkable inhibitory effect on tumor formation[44]. STAT3 activation and subsequent dimer formation initiates nuclear translocation of STAT3 for the regulation of target gene transcription[9]. The Jaspar transcriptional interaction website predicted that STAT3 could potentially bind to the PyCR1 promoter region. STAT3 promoted PYCR1 gene and protein level, meanwhile increased the enrichment of STAT3 protein in the PYCR1 promoter region. We further showed that STAT3 interacted with the promoter of PYCR1. Knock-down of PYCR1 inhibited Epithelial to mesenchymal transition of bladder cancer, and simultaneously mitigated the carcinogenic effects of STAT3. 
Here, we tested STAT3 and SENP3 expression in bladder cancer tissues and examined its connection and molecular mechanism in bladder cancer. We found that SENP3 induced STAT3 protein level and p-STAT3 translocating into nuclear through deSUMOylation of STAT3. Nuclear STAT3, as a transcriptional activity factor, promoted PYCR1 gene and protein level by interacting with the promoter of PYCR1. Our results lend novel insights into the regulation of STAT3, which has key roles in bladder cancer progression and may aid in identifying new biomarkers or targeted therapies for bladder cancer

\section{Declarations}

\section{Conflict of interest}

The authors declare that they have no conflict of interest.

\section{Funds}

This study was funded by Hunan Provincial Health Commission Project(No. 20200044); the Project of Hunan Provincial Department of Education (No.20C1174); Changsha Municipal Natural Science Foundation (No.kq2014096).

\section{Authors' contributions}

Li Zhuo and Jian Liu conceived and designed the study. Huifeng Fu, Yuanwei Li and Qaing Lu performed experiments. Wei Song collected the data. Jian Liu and Huifeng Fu interpreted the data. Mingqiang Zeng and Li Zhuo analyzed the data and wrote the manuscript. All authors reviewed the results and approved the final version of the manuscript.

\section{References}

1. Gupta, B. and N. Kumar, Worldwide incidence, mortality and time trends for cancer of the oesophagus. Eur J Cancer Prev, 2017. 26(2): p. 107-118.

2. Hung, C.F., C.K. Yang, and Y.C. Ou, Urologic cancer in Taiwan. Jpn J Clin Oncol, 2016. 46(7): p. 605-9.

3. Wong, M.C.S., et al., The global epidemiology of bladder cancer: a joinpoint regression analysis of its incidence and mortality trends and projection. Sci Rep, 2018. 8(1): p. 1129.

4. Chavan, S., et al., International variations in bladder cancer incidence and mortality. Eur Urol, 2014. 66(1): p. 59-73.

5. Lapitan, M.C.M., J.D. Cody, and A. Mashayekhi, Open retropubic colposuspension for urinary incontinence in women. Cochrane Database Syst Rev, 2017. 7: p. CD002912.

6. Guo, C.C., et al., Assessment of Luminal and Basal Phenotypes in Bladder Cancer. Sci Rep, 2020. 10(1): p. 9743.

7. Ng, K., et al., Urinary biomarkers in bladder cancer: A review of the current landscape and future directions. Urol Oncol, 2021. 39(1): p. 41-51. 
8. Ma, S., et al., Melatonin derivatives combat with inflammation-related cancer by targeting the Main Culprit STAT3. Eur J Med Chem, 2021. 211: p. 113027.

9. Liu, Y., et al., STAT3 and its targeting inhibitors in osteosarcoma. Cell Prolif, 2021. 54(2): p. e12974.

10. Peng, W., et al., miR-4500 suppresses cell proliferation and migration in bladder cancer via inhibition of STAT3/CCR7 pathway. J Cell Biochem, 2019.

11. Lai, S.C., et al., DNMT3b/OCT4 expression confers sorafenib resistance and poor prognosis of hepatocellular carcinoma through IL-6/STAT3 regulation. J Exp Clin Cancer Res, 2019. 38(1): p. 474.

12. Cocchiola, R., et al., STAT3 Post-Translational Modifications Drive Cellular Signaling Pathways in Prostate Cancer Cells. Int J Mol Sci, 2019. 20(8).

13. Huang, G., et al., STAT3 phosphorylation at tyrosine 705 and serine 727 differentially regulates mouse ESC fates. Stem Cells, 2014. 32(5): p. 1149-60.

14. Lau, W.W., et al., Interleukin-6 autocrine signaling mediates melatonin MT(1/2) receptor-induced STAT3 Tyr(705) phosphorylation. J Pineal Res, 2012. 52(4): p. 477-89.

15. Ge, Q., et al., miR-4324-RACGAP1-STAT3-ESR1 feedback loop inhibits proliferation and metastasis of bladder cancer. Int J Cancer, 2019. 144(12): p. 3043-3055.

16. Zhou, Z., et al., SUMOylation and SENP3 regulate STAT3 activation in head and neck cancer. Oncogene, 2016. 35(45): p. 5826-5838.

17. Zhang, Y., et al., SENP3 protects H9C2 cells from apoptosis triggered by H/R via STAT3 pathway. Eur Rev Med Pharmacol Sci, 2018. 22(9): p. 2778-2786.

18. Hindupur, S.V., et al., STAT3/5 Inhibitors Suppress Proliferation in Bladder Cancer and Enhance Oncolytic Adenovirus Therapy. Int J Mol Sci, 2020. 21(3).

19. Chang, H.M. and E.T.H. Yeh, SUMO: From Bench to Bedside. Physiol Rev, 2020. 100(4): p. 1599-1619.

20. Kunz, K., T. Piller, and S. Muller, SUMO-specific proteases and isopeptidases of the SENP family at a glance. J Cell Sci, 2018. 131(6).

21. Tokarz, P. and K. Wozniak, SENP Proteases as Potential Targets for Cancer Therapy. Cancers (Basel), 2021. 13(9).

22. Bhagwat, N.R., et al., SUMO is a pervasive regulator of meiosis. Elife, 2021. 10.

23. Li, Y.J., et al., Allosteric Inhibition of Ubiquitin-like Modifications by a Class of Inhibitor of SUMOActivating Enzyme. Cell Chem Biol, 2019. 26(2): p. 278-288 e6.

24. Long, X., et al., The Critical Roles of the SUMO-Specific Protease SENP3 in Human Diseases and Clinical Implications. Front Physiol, 2020. 11: p. 558220.

25. Weijin, F., et al., The clinical significance of PYCR1 expression in renal cell carcinoma. Medicine (Baltimore), 2019. 98(28): p. e16384.

26. Song, W., et al., Dysregulation of USP18/FTO/PYCR1 signaling network promotes bladder cancer development and progression. Aging (Albany NY), 2021. 13(3): p. 3909-3925.

27. Christensen, E.M., et al., In crystallo screening for proline analog inhibitors of the proline cycle enzyme PYCR1. J Biol Chem, 2020. 295(52): p. 18316-18327. 
28. Wang, D., et al., PYCR1 promotes the progression of non-small-cell lung cancer under the negative regulation of miR-488. Biomed Pharmacother, 2019. 111: p. 588-595.

29. Antoni, S., et al., Bladder Cancer Incidence and Mortality: A Global Overview and Recent Trends. Eur Urol, 2017. 71(1): p. 96-108.

30. Bray, F., et al., Global cancer statistics 2018: GLOBOCAN estimates of incidence and mortality worldwide for 36 cancers in 185 countries. CA Cancer J Clin, 2018. 68(6): p. 394-424.

31. Nakayama, M., et al., Impact of sex difference on survival of bladder cancer: A population-based registry data in Japan. Int J Urol, 2019. 26(6): p. 649-654.

32. Wen, Z., Z. Zhong, and J.E. Darnell, Jr., Maximal activation of transcription by Stat1 and Stat3 requires both tyrosine and serine phosphorylation. Cell, 1995. 82(2): p. 241-50.

33. Busch, S., et al., mTOR mediates human trophoblast invasion through regulation of matrixremodeling enzymes and is associated with serine phosphorylation of STAT3. Exp Cell Res, 2009. 315(10): p. 1724-33.

34. Yang, J., et al., Reversible methylation of promoter-bound STAT3 by histone-modifying enzymes. Proc Natl Acad Sci U S A, 2010. 107(50): p. 21499-504.

35. Zhong, Z., Z. Wen, and J.E. Darnell, Jr., Stat3: a STAT family member activated by tyrosine phosphorylation in response to epidermal growth factor and interleukin-6. Science, 1994. 264(5155): p. 95-8.

36. Jiang, X., et al., Overexpressed coiled-coil domain containing protein 8 (CCDC8) mediates newly synthesized HIV-1 Gag lysosomal degradation. Sci Rep, 2020. 10(1): p. 11416.

37. Ulane, C.M., et al., STAT3 ubiquitylation and degradation by mumps virus suppress cytokine and oncogene signaling. J Virol, 2003. 77(11): p. 6385-93.

38. Yeh, E.T., L. Gong, and T. Kamitani, Ubiquitin-like proteins: new wines in new bottles. Gene, 2000. 248(1-2): p. 1-14.

39. Rosonina, E., et al., Regulation of transcription factors by sumoylation. Transcription, 2017. 8(4): p. 220-231.

40. Lao, Y., et al., DeSUMOylation of MKK7 kinase by the SUMO2/3 protease SENP3 potentiates lipopolysaccharide-induced inflammatory signaling in macrophages. J Biol Chem, 2018. 293(11): p. 3965-3980.

41. Cheng, J., et al., Upregulation of SENP3/SMT3IP1 promotes epithelial ovarian cancer progression and forecasts poor prognosis. Tumour Biol, 2017. 39(3): p. 1010428317694543.

42. Chen, S., et al., SIRT3 regulates cancer cell proliferation through deacetylation of PYCR1 in proline metabolism. Neoplasia, 2019. 21(7): p. 665-675.

43. Kuo, M.L., et al., PYCR1 and PYCR2 Interact and Collaborate with RRM2B to Protect Cells from Overt Oxidative Stress. Sci Rep, 2016. 6: p. 18846.

44. Du, S., et al., PYCR1 promotes bladder cancer by affecting the Akt/Wnt/beta-catenin signaling. J Bioenerg Biomembr, 2021. 53(2): p. 247-258. 


\section{Supplementary Materials}

Supplementary Materials are not available with this version.

\section{Figures}

A

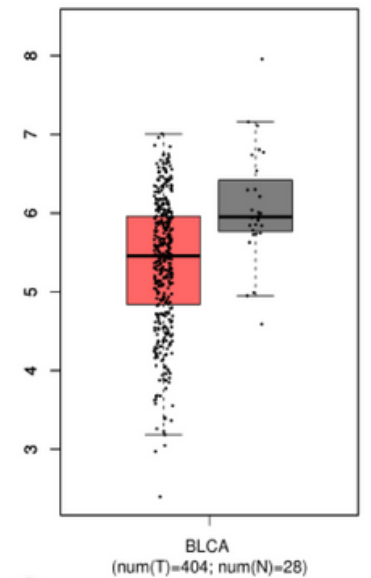

C

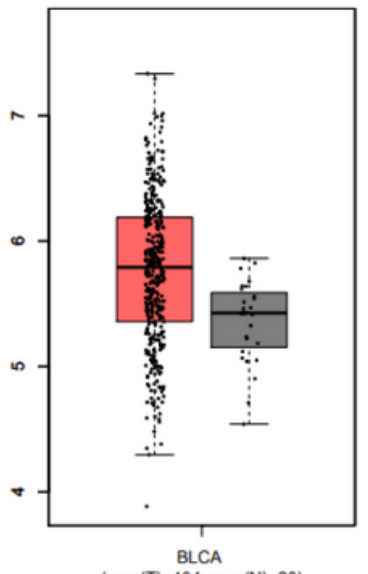

E

(num $(\mathrm{T})=404 ; \operatorname{num}(\mathrm{N})=28$ )

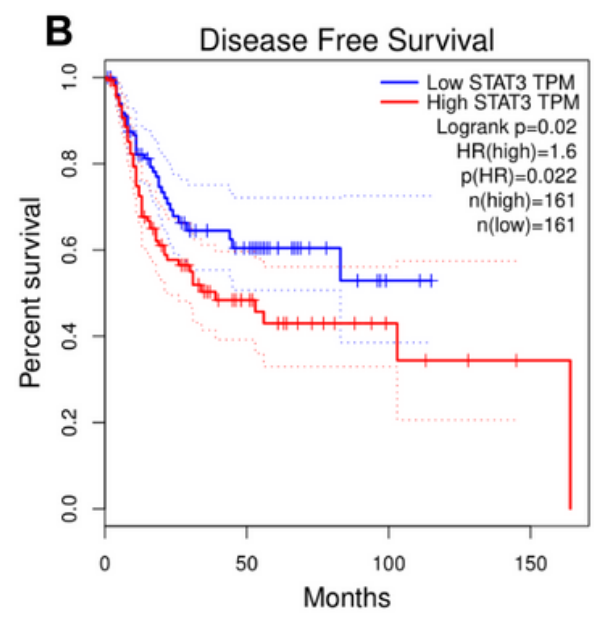

D

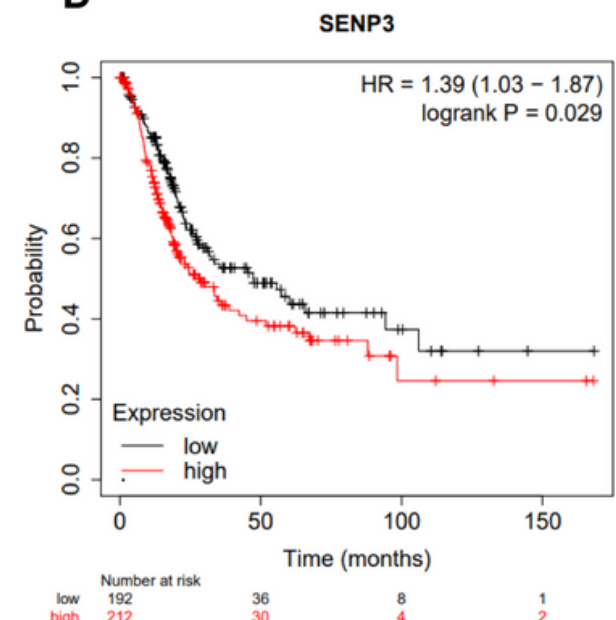
$\begin{array}{ccc}\text { low } & 192 \\ \text { high } & 212 & 36\end{array}$
$\mathbf{F}$

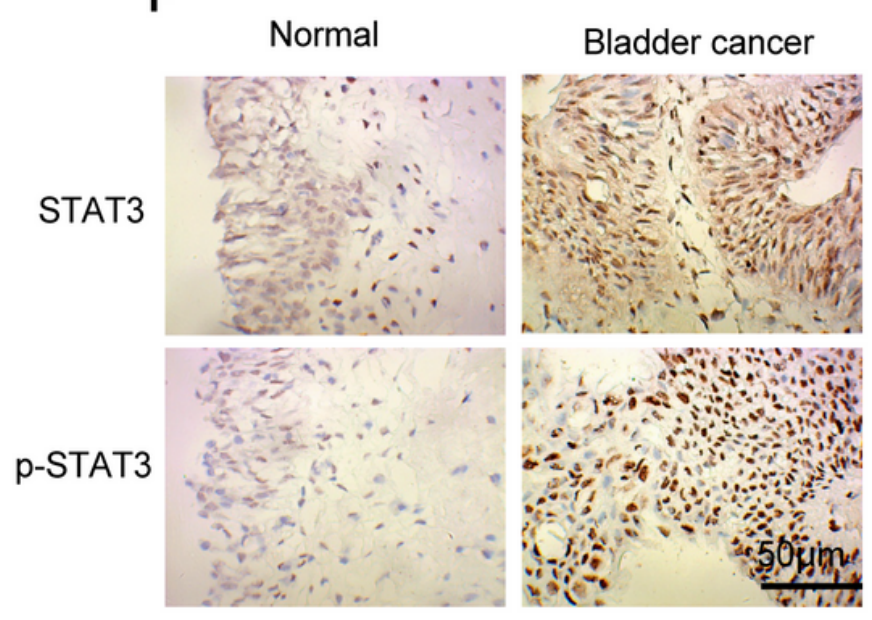

G

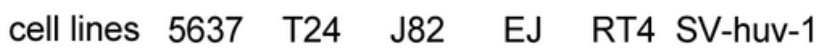

SENP3

STAT3

p-STAT3

$\beta$-actin
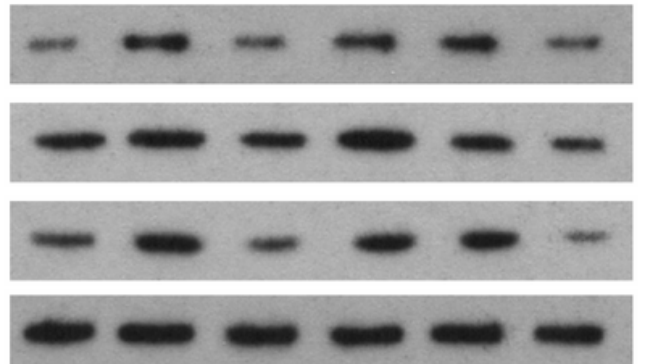

$\begin{array}{llllllllllllllll}\text { T1 } & \mathrm{N} 1 & \mathrm{~T} 2 & \mathrm{~N} 2 & \mathrm{~T} 3 & \mathrm{~N} 3 & \mathrm{~T} 4 & \mathrm{~N} 4 & \mathrm{~T} 5 & \mathrm{~N} 5 & \mathrm{~T} 6 & \mathrm{~N} 6 & \mathrm{~T} 7 & \mathrm{~N} 7 & \mathrm{~T} 8 & \mathrm{~N} 8\end{array}$

SENP3

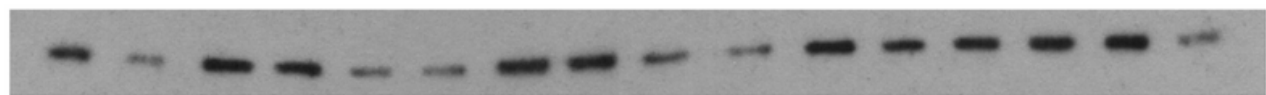

STAT3

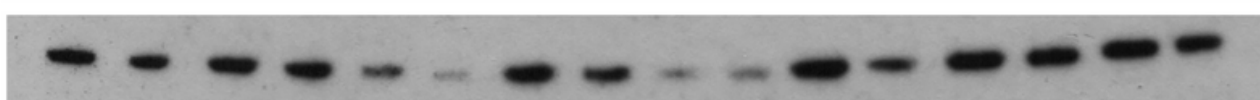

p-STAT3

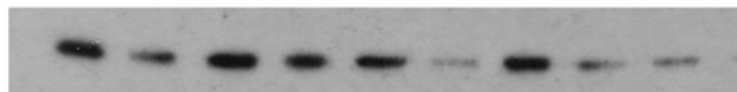

$---\infty$

$\beta$-actin

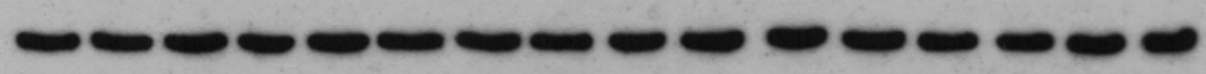

\section{Figure 1}

SENP3 protein level correlates with protein expression of STAT3 and p-STAT3 in bladder cancer (A) Differences of STAT3 between bladder cancer tissues and adjacent normal tissues, determined by bioinformatics analysis. (B) Disease free survival with low and high STAT3 IPM. (C) SENP3 level between 
bladder cancer tissues and adjacent normal tissues, determined by bioinformatics analysis. (D) Probability of low and high expression. (E) SENP3, STAT3 and p-STAT3 protein levels in bladder cancer tissues and adjacent normal tissues, as measured by western blot. (F) Expression of STAT3 and p-STAT3 in bladder cancer tissues and adjacent normal tissues by immunohistochemistry [scale bar, $50 \mu \mathrm{m}$ ]. (G) SENP3, STAT3 and p-STAT3 protein levels in 5637, T24, J82, EJ, RT4 and SV-huv-1 cells.
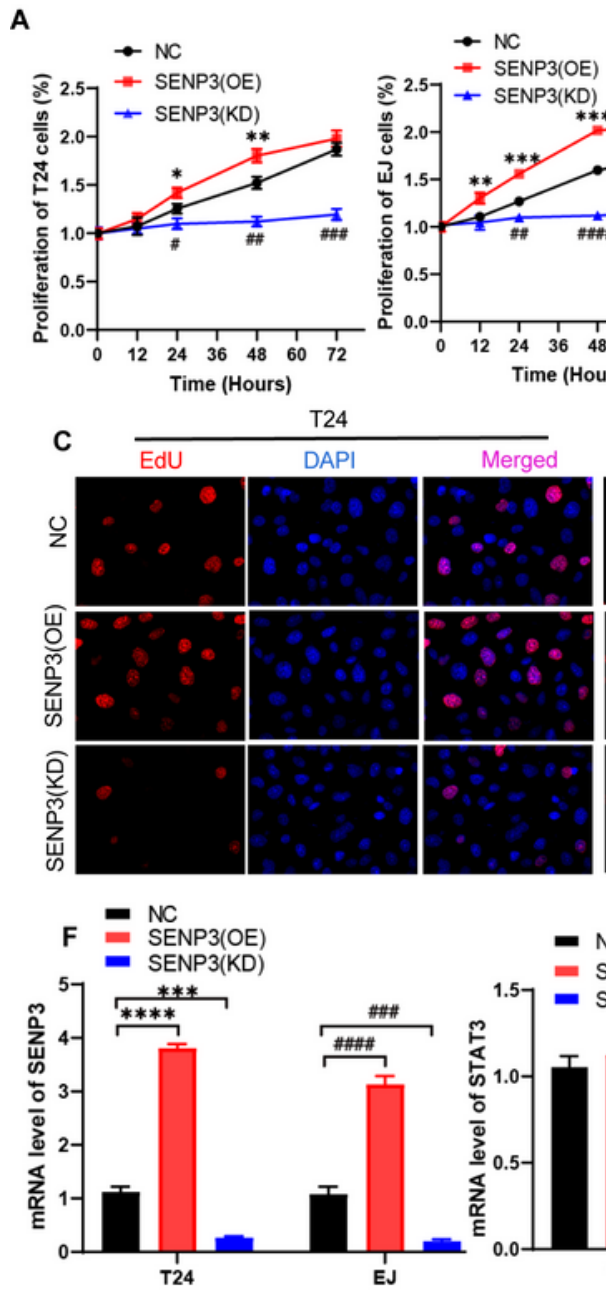

H

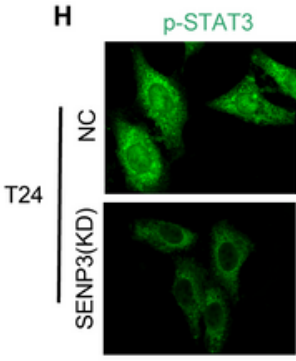

DAPI
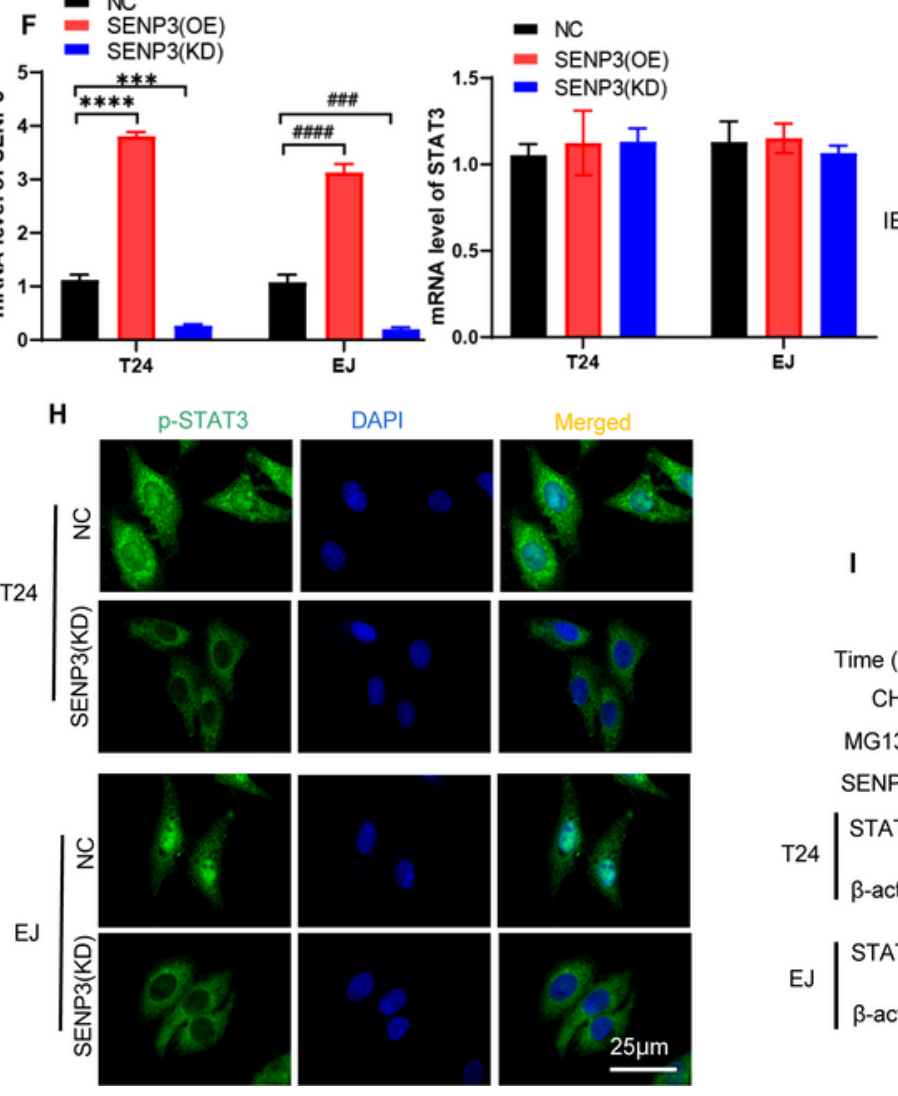

B
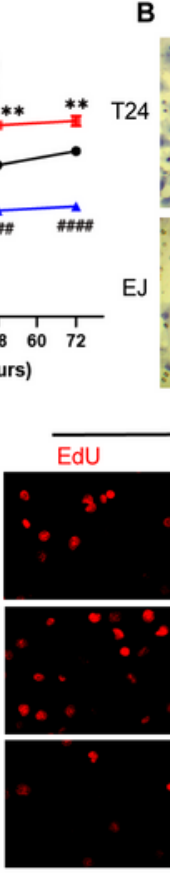

EJ

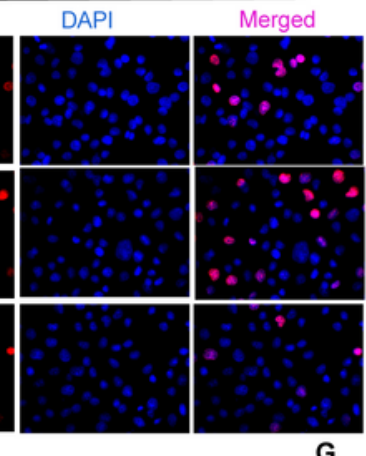

G
SENP3(OE)

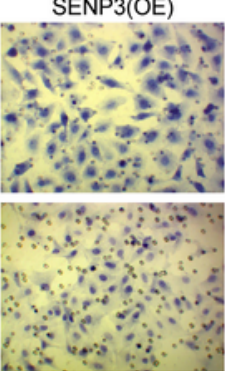

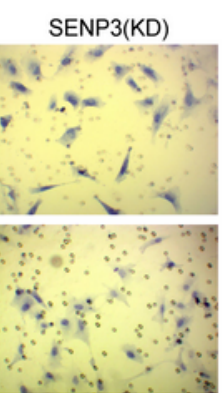

E
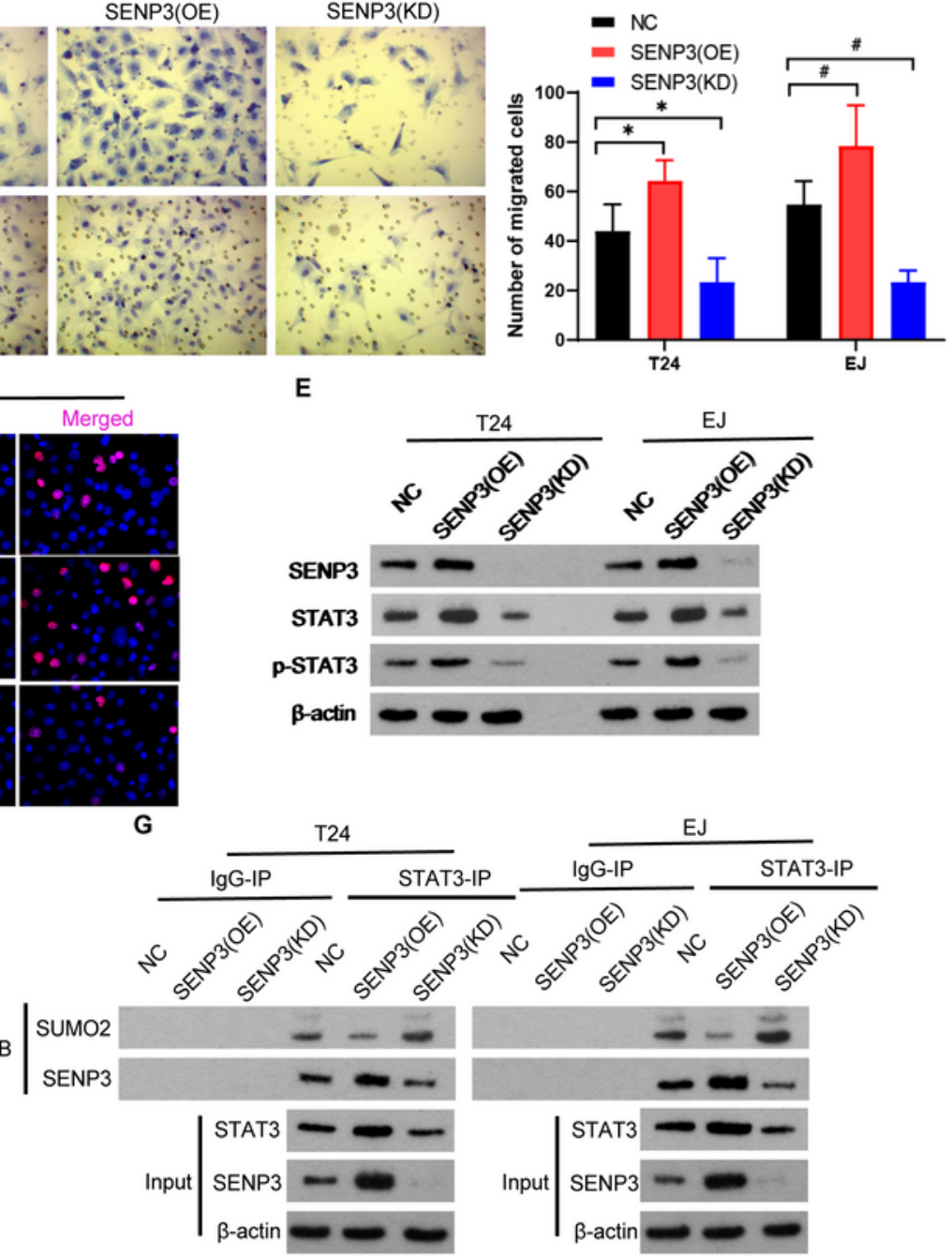

Figure 2

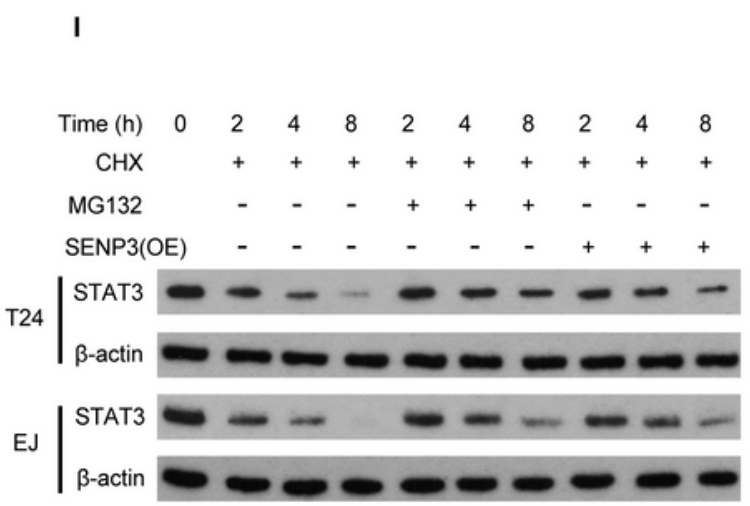


SENP3 promotes proliferation and deSUMOylation of STAT3 T24 and EJ cells were transfected plasmids with overexpression of SENP3 (SENP3(OE)) and knock-down of SENP3 (SENP3(KD)). (A) Cell proliferation assay in vitro in NC, SENP3(OE), SENP3(KD) T24 and EJ cells. [mean \pm S.D. (error bars), $n=$

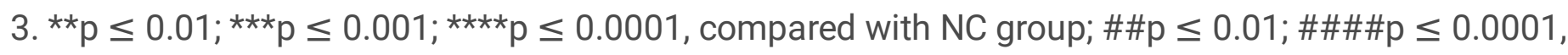
compared with NC group, two-way analysis of variance]. (B) Left: Cell invasion determined by trans well staining in SENP3 overexpression or knockdown T24 and EJ cells. [scale bar, $25 \mu \mathrm{m}$ ]. right: quantitative analysis of immunohistochemistry for positive trans well staining. [mean \pm S.D. (error bars), $n=3 .{ }^{*} p \leq$ 0.05 , compared in T24 cells; \#p $\leq 0.05$, compared in EJ cells, two-way analysis of variance] (C) EDU positive cells in NC, SENP3(OE), SENP3(KD) T24 and EJ cells determined by Confocal

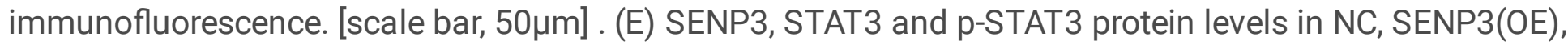
SENP3(KD) T24 and EJ cells, as measured by western blot. (F) The mRNA level of SENP3 and STAT3 measured by qPCR. (G) Co-immunoprecipitation (co-IP) of endogenous SENP3 with STAT3 and its SUMO2. (H) Abundance of p-STAT3 protein in in NC, SENP3(OE) T24 and EJ cells. (J) T24 cells were transfected with the indicated constructs and treated for the indicated times with CHX and MG132, whole cells were collected and STAT3 protein level was determined by western blot. All experiments were performed in triplicates. 

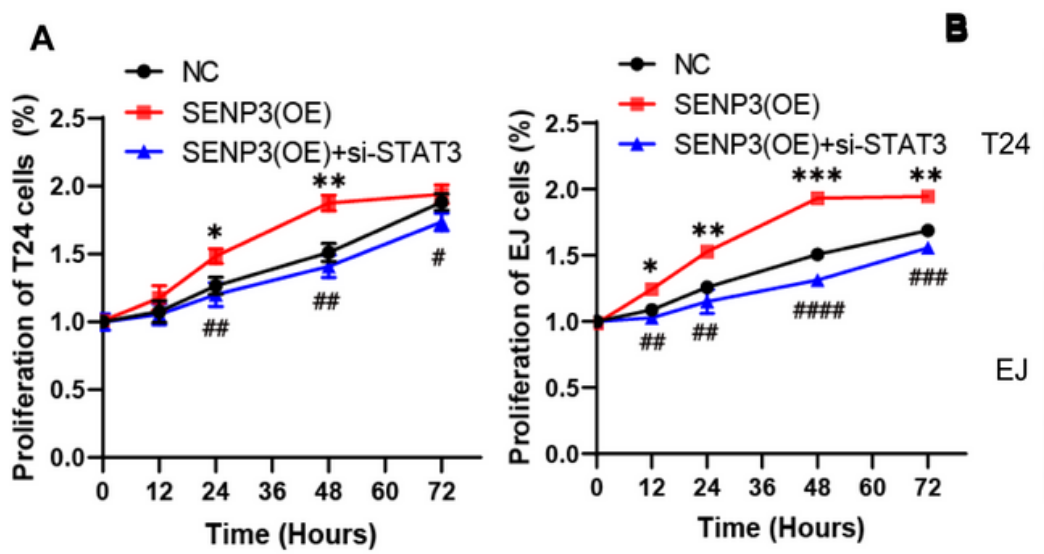

C
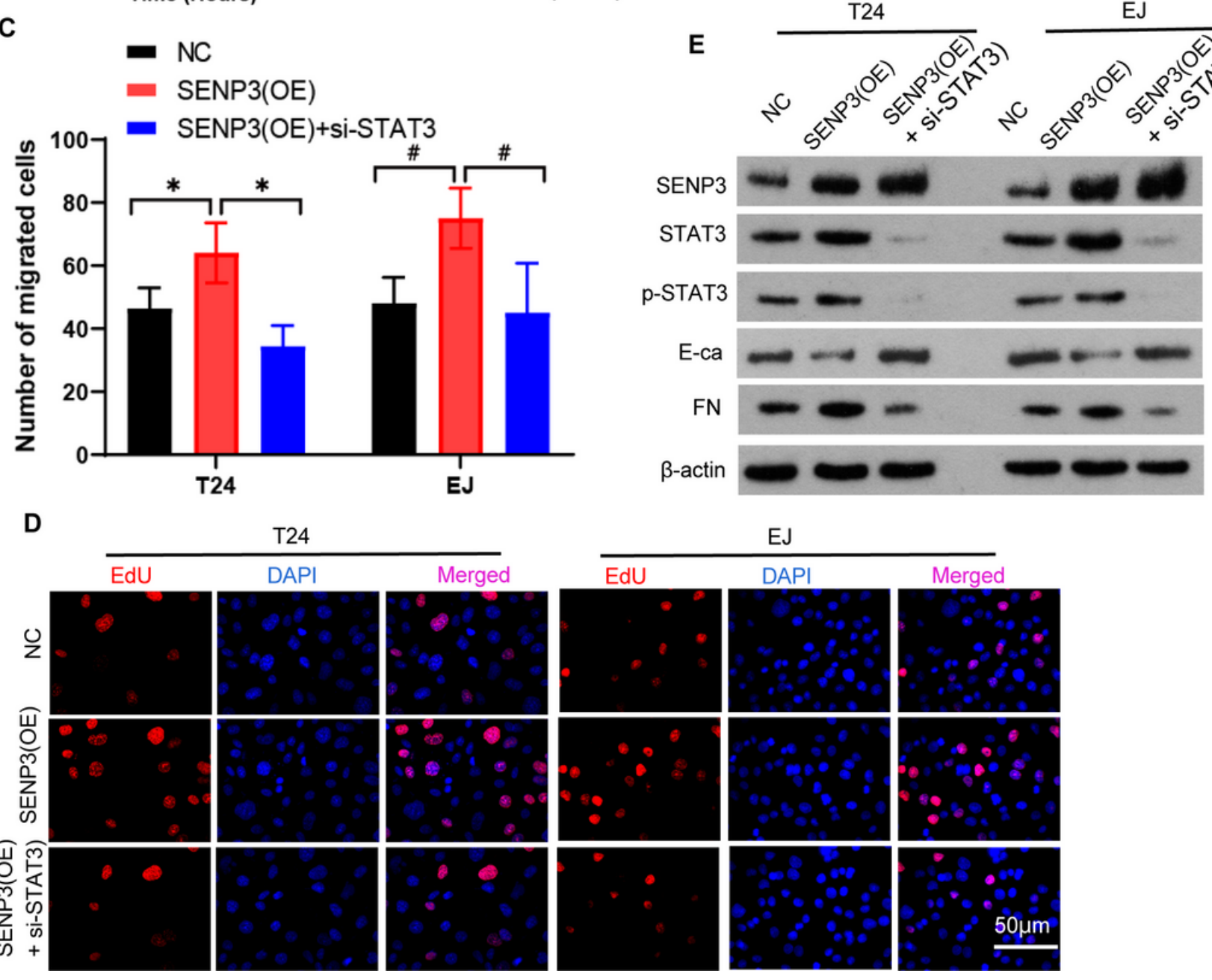

T24

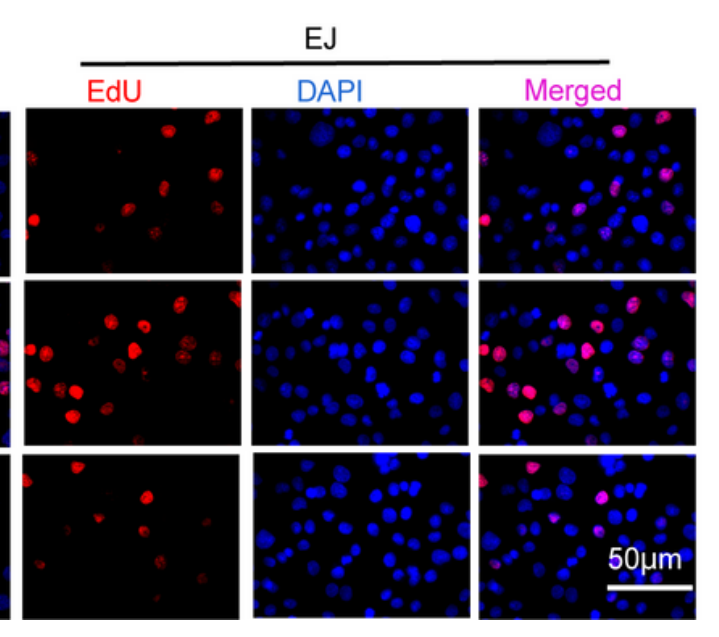

Figure 3

STAT3 mitigates the cancer-promoting effect of SENP3 (A) Overexpression of SENP3(SENP3(OE)) T24 and EJ cells were transfected with STAT3 siRNAs (SENP3(OE)+si-STAT3). Cell proliferation were determined by CCK8 essay in NC, SENP3(OE), SENP3(OE)+si-STAT3 T24 and EJ cells. [mean \pm S.D. (error

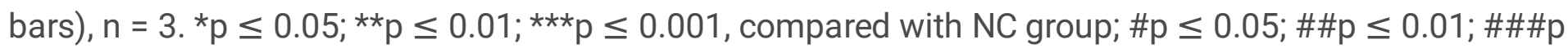
$\leq 0.001$; \#\#\#\# $\leq 0.0001$, compared with SENP(OE) group, two-way analysis of variance]. (B) Cell invasion determined by trans well staining in NC, SENP3(OE), SENP3(OE)+si-STAT3 T24 and EJ cells. (C) Quantitative analysis of immunohistochemistry for positive trans well staining. [mean \pm S.D. (error bars), $n=3 .{ }^{*} \mathrm{p} \leq 0.05$, compared in T24 cells; \#p $\leq 0.05$, compared in EJ cells, two-way analysis of variance]. 
(D) Abundance of the indicated protein was analyzed by Western blotting. (E) EDU positive cells in NC, SENP3(OE), SENP3(OE)+si-STAT3 T24 and EJ cells determined by Confocal immunofluorescence. All experiments were performed in triplicates.
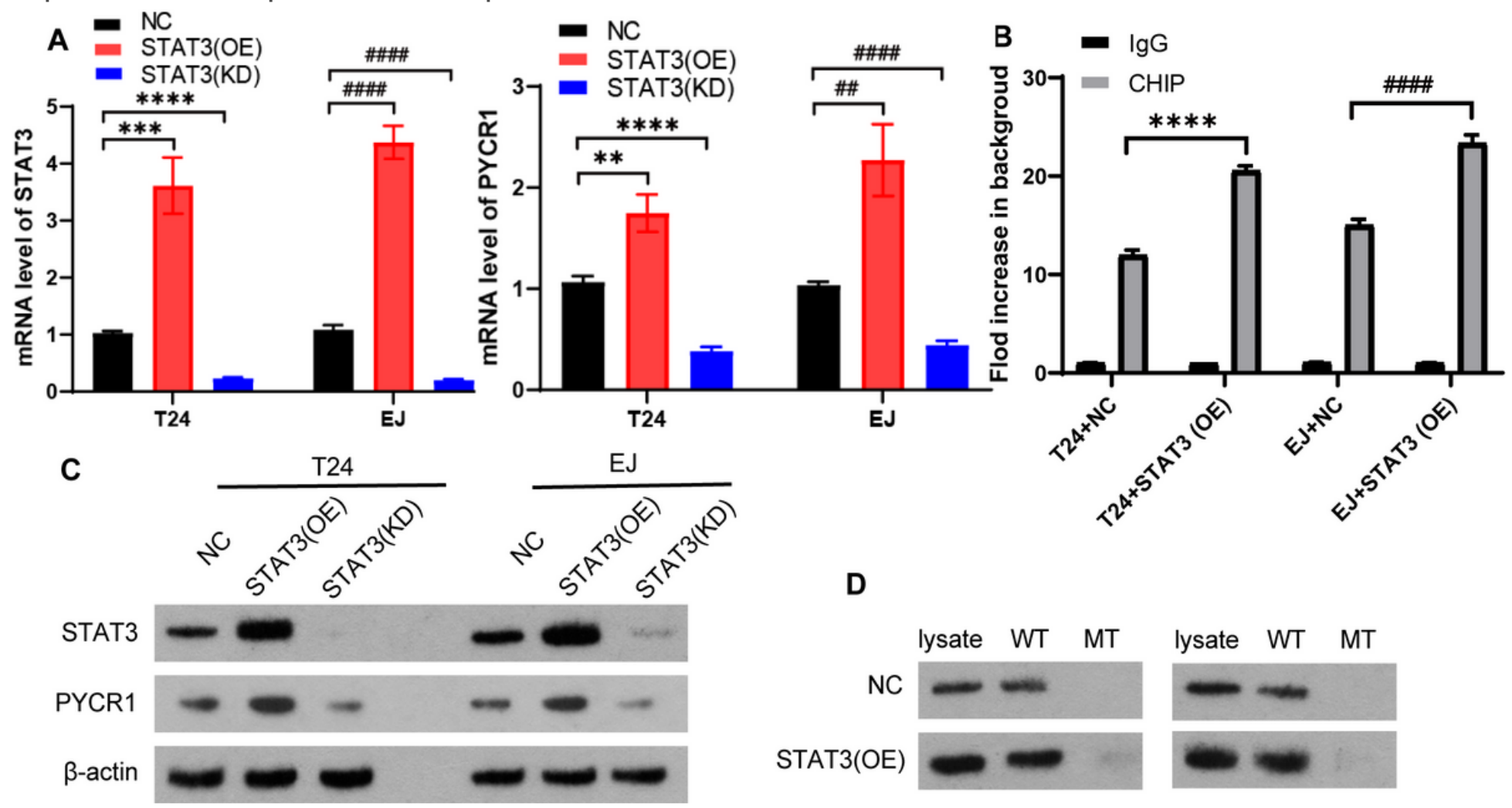

Figure 4

STAT3 promotes gene and protein levels of PYCR1 by binding to promoter of PYCR1. T24 and EJ cells were transfected plasmids with overexpression of STAT3 (STAT3(OE)) and knock-down of STAT3 (STAT3(KD)). (A) The mRNA level of SENP3 and STAT3 measured by qPCR in NC, STAT3(OE), STAT3(KD) T24 and EJ cells. All data in this figure are represented as mean \pm SD. ${ }^{*} P<0.05$, compared in T24 cells; \# $\mathrm{P}<0.05$, compared in EJ cells. (B) The regulation of STAT3 on promoter region of ZNF667, determined by ChIP assay. [mean \pm S.D. (error bars), $n=4 .{ }^{* * \star *} p \leq 0.0001$, compared in T24 cells; \#\#\#\#p $\leq .0001$, compared in EJ cells, two-way analysis of variance] (C) STAT3 and PYCR1 protein level of NC, STAT3(OE), STAT3(KD) T24 and EJ cells. (D) The high score region of the predicted binding sites between PYCR1 promoter and STAT3 protein by DNA-affinity precipitation assay (DAPA), the oligonucleotide DNA probe containing the above binding region and the corresponding mutation probe were designed for DAPA detection. All experiments were performed in triplicates. 

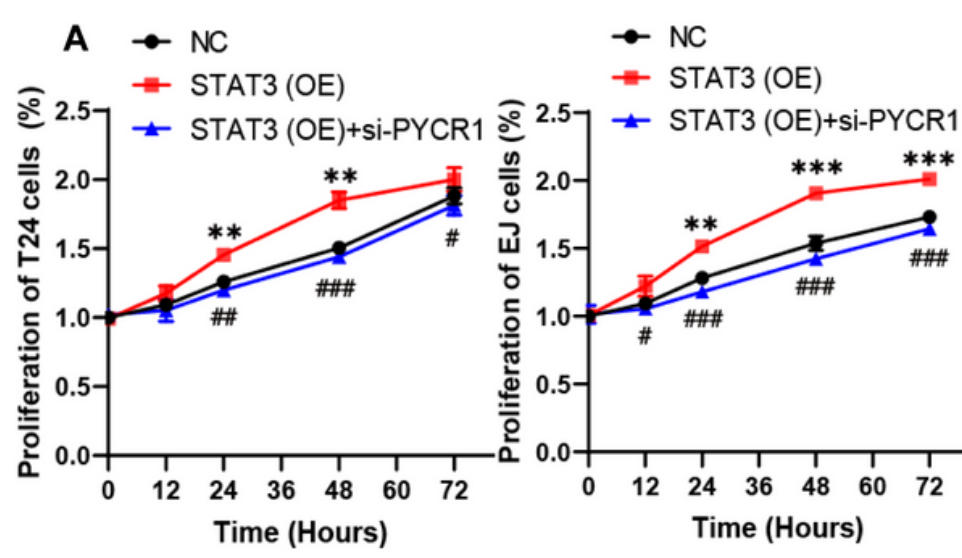

C

- NC

- STAT3 (OE)

- STAT3(OE)+si-PYCR1

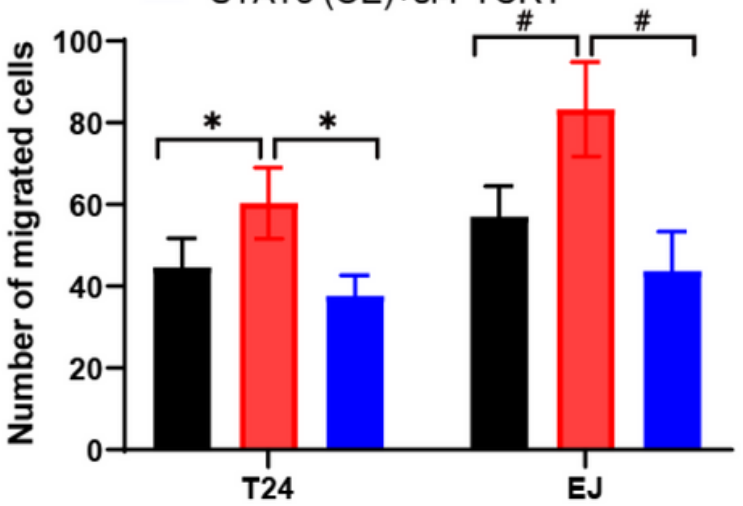

D

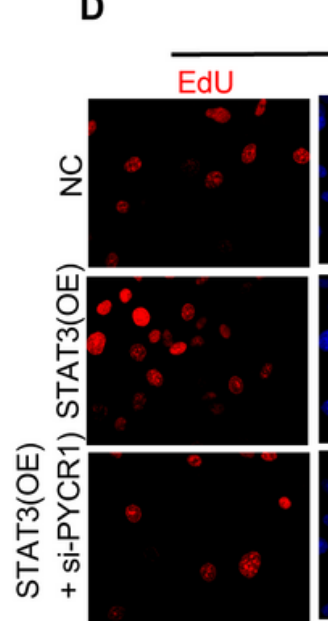

T24
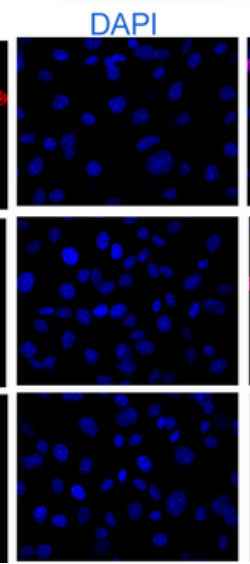

B

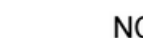

NC

STAT3(OE) STAT3(OE)+si-PYCR1

T24

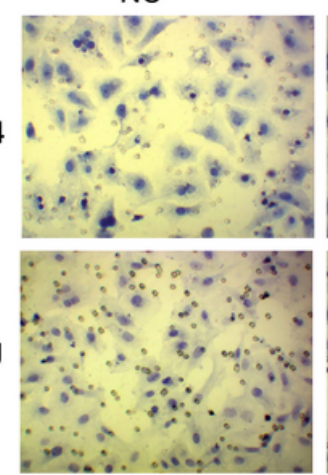

T24

E

STAT3

p-STAT3

PYCR1

E-ca

FN

$\beta$-actin

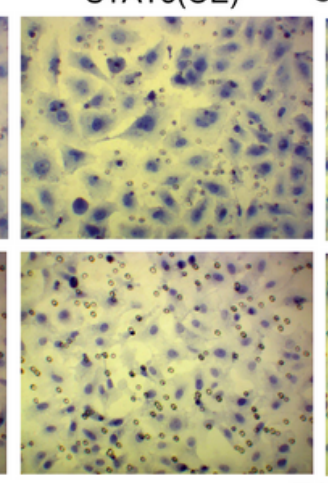

EJ
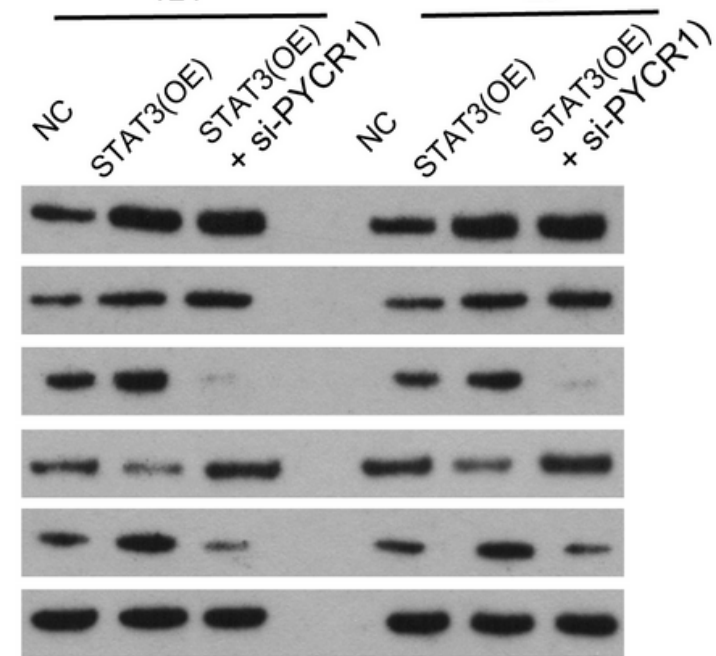
$25 \mu \mathrm{m}$

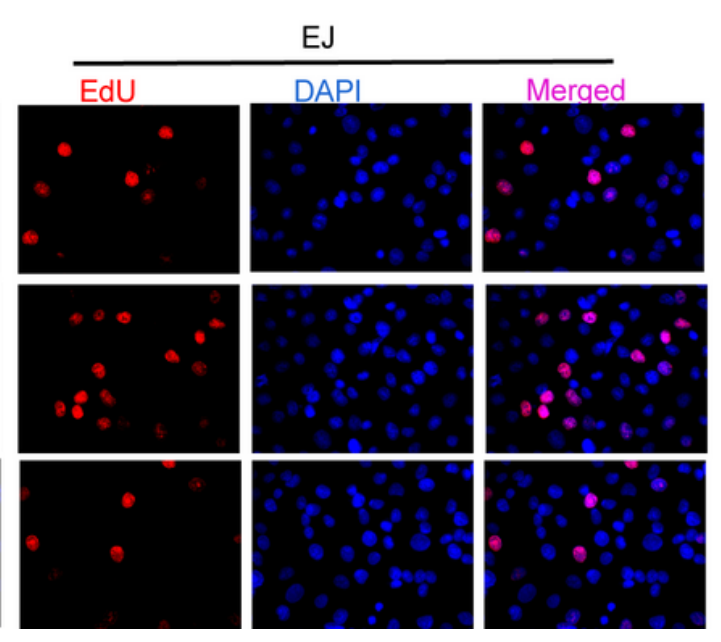

Figure 5

PYCR1 mitigates the carcinogenic effect of STAT3 (A) Overexpression of STAT3(STAT3(OE)) T24 and EJ cells were transfected with PYCR1 siRNAs (STAT3(OE)+si-PYCR1). Cell proliferation were determined by CCK8 essay in NC, SENP3(OE), SENP3(OE)+si-STAT3 T24 and EJ cells. [mean \pm S.D. (error bars), $\mathrm{n}=3$. ${ }^{* *} \mathrm{p} \leq 0.01 ;{ }^{* *} \mathrm{p} \leq 0.001$, compared with NC group; $\# \mathrm{p} \leq 0.05 ; \# \# \mathrm{p} \leq 0.01 ; \# \# \# \mathrm{p} \leq 0.001$, compared with STAT3(OE) group, two-way analysis of variance]. (B) Cell invasion determined by trans well staining in NC, STAT3(OE), STAT3(OE)+si-PYCR1 T24 and EJ cells. (C) Quantitative analysis of immunohistochemistry for positive trans well staining. All data in this figure are represented as mean \pm 
SD. * $\mathrm{P}<0.05$, compared in T24 cells; \# $\mathrm{P}<0.05$, compared in EJ cells. (D) Abundance of the indicated protein was analyzed by Western blotting. (E) EDU positive cells in in NC, SENP3(OE), SENP3(OE)+siSTAT3 T24 and EJ cells determined by Confocal immunofluorescence. All experiments were performed in triplicates.

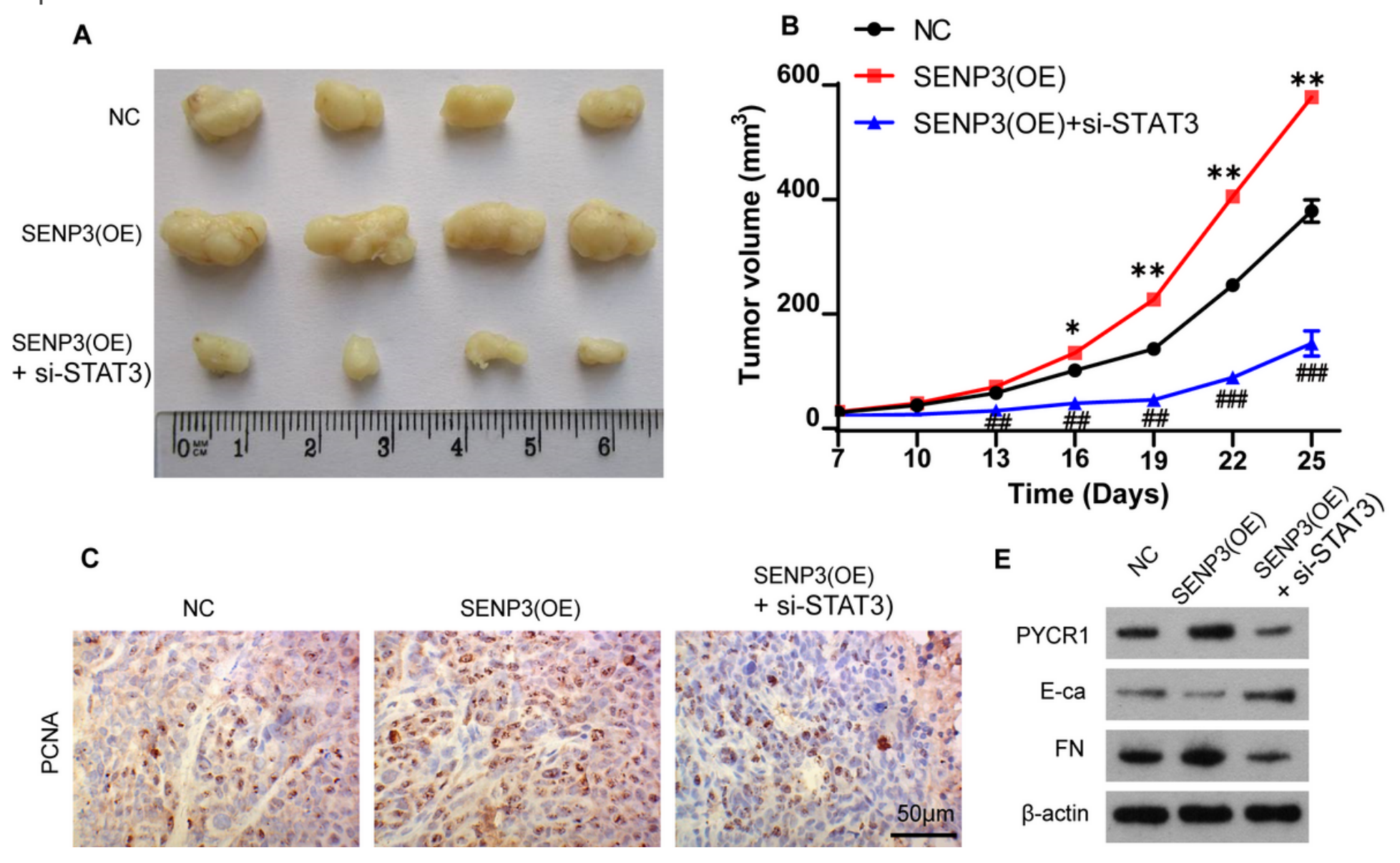

Figure 6

SENP3 promotes tumor proliferation by upregulating STAT3 in vivo (A) A subcutaneous transplantation tumor model in nude BALB/c mice was established using the human bladder cancer cell line T24. The mice were divided into NC, SENP3(OE) and SENP3(OE)+si-STAT3 groups with 5 mice per group. (B) The growth curve of tumor volumes. [mean \pm S.D. (error bars), $n=4$. ${ }^{\star} p \leq 0.05 ;{ }^{*} p \leq 0.01$, compared with NC group; \#\#p $\leq 0.01$; \#\#\# $\leq$ 0.001, compared with STAT3(OE) group, two-way analysis of variance]. (C) PCNA positive cells in subcutaneous transplantation tumor isolates by immunohistochemistry. [scale bar, $50 \mu \mathrm{m}]$. (D) PYCR1, E-ca and FN protein level of NC, SENP3(OE) and SENP3(OE)+si-STAT3 mice transplantation tumor: 